\title{
The evolutionary history of Drosophila buzzatii. XVII. Double mating and sperm predominance
}

\author{
A Barbadilla*, JE Quezada-Díaz, A Ruiz, \\ $M$ Santos, A Fontdevila \\ Universidad Autónoma de Barcelona, Departamento de Genética y Microbiología, \\ 08193 Bellaterra, Barcelona, Spain
}

(Received 22 January 1990; accepted 30 January 1991)

\begin{abstract}
Summary - Sperm predominance in males and double mating in females have been studied in 2 stocks of the cactophilic species Drosophila buzzatii. The relationship between double mating and total productivity of females was also ascertained. Our results show high values of sperm predominance and double mating. Moreover, female productivity is increased with a second mate. These results are discussed in relation to the mating strategy of this species.
\end{abstract}

Drosophila buzzatti / sperm predominance / double mating / mate strategy / total productivity

Résumé - Histoire évolutive de Drosophila buzzatii. XVII. Accouplement double et prédominance du sperme. On a étudié la prédominance du sperme chez les mâles et le double accouplement chez les femelles dans 2 souches de l'espèce cactophile Drosophila buzzatii. La relation entre le double accouplement et la productivité totale des femelles a été aussi recherchée. Nos résultats montrent des valeurs élevées pour la prédominance du sperme et pour le double accouplement. De plus, on constate que la productivité des femelles est augmentée par un deuxième accouplement. Ces résultats sont discutés par rapport à la stratégie d'accouplement de cette espèce.

Drosophila buzzatii / prédominance du sperme / accouplement double / stratégie d'accouplement / productivité totale

\section{INTRODUCTION}

Multiple mating is a widespread phenomenon among insect females (Thornhill and Alcock, 1983; Smith, 1984; Ridley, 1988). If the sperm of the first male is not exhausted before female remating, then sperm competition occurs in the storage organs of the female between the sperms of different origin (Parker, 1970, 1984). Sperm predominance, usually that of the last mated male, is the general result of

\footnotetext{
* Correspondence and reprints
} 
this competition. When there are genetic differences in the degree of predominance, sperm predominance may result in sexual selection. Prout and Bundgaard (1977) showed theoretically how this type of selection could maintain a population in stable equilibrium for 2 alleles. The mating strategies of many species are determined by the importance of sperm predominance in males and multiple mating in females (Smith, 1984).

Several experimental studies have been carried out to ascertain the degree of sperm predominance in Drosophila (Gromko et al, 1984). In the present work, we have studied sperm predominance in the cactophilic species Drosophila buzzatii. In addition, the frequency of double mating and its influence on the total female productivity were determined. $D$ buzzatii belongs to the repleta group of Drosophila and several aspects of its ecology and mating behaviour have been extensively studied in our laboratory (Ruiz et al, 1986, Santos et al, 1988, 1989).

\section{MATERIALS AND METHODS}

Two stocks were used in this experiment. One, the wild type stock, was derived from a natural population collected at Carboneras, Almería (SE Spain), in May 1986. The other stock was homozygous for the sex-linked recessive white mutant which arose spontaneously and was subsequently isolated in our laboratory in April 1983. Since no attempt was made to randomize the genetic background of the 2 stocks, they might differ at many loci and the mutant white was merely a genetic marker.

The experimental procedure was similar to that of Turner and Anderson (1984). The crosses performed are shown in table I. The w/+ females and the w/Y males were the hybrid offspring from the 2 parental stocks. The experiment began with the first cross. Five to 6 -d old virgin females were crossed individually with 2 males of the same age. The crosses were carried out in $2 \times 8 \mathrm{~cm}$ vials with $\mathrm{ca} 8 \mathrm{~cm}^{3}$ of food medium. After $24 \mathrm{~h}$ the males were discarded. Two d later the second cross was made, also with 2 males per female. After $2 \mathrm{~d}$, the males were discarded and each female was transferred daily for 11 consecutive days without etherization into vials with fresh food. Thereafter, new transfers were made at 2-d intervals.

Table I. Mating types performed, classified according to the female genotype and the sequence of male genotypes. $n=$ No of females.

\begin{tabular}{lcccc}
\hline Group & Female & 1st male & 2nd male & $\mathrm{n}$ \\
\hline 1a & w/w & w/Y & $+/ Y$ & 50 \\
1b & w/w & $+/ Y$ & w/Y & 50 \\
2a & w/t & w/Y & $+/ Y$ & 50 \\
2b & w/t & $+/ Y$ & w/Y & 50 \\
3a (control) & w/w & w/Y & & 25 \\
3b (control) & w/w & $+/ Y$ & & 25 \\
4a (control) & w/t & w/Y & & 25 \\
4b (control) & w/t & $+/ Y$ & & 25 \\
\hline
\end{tabular}


All the individuals were grown at nearly optimal density (4-5 larvae per $\mathrm{cm}^{3}$ of medium). A modified formula of David's killed-yeast Drosophila medium (David, 1962) was used as food. The flies were kept at $23^{\circ} \mathrm{C}$. The offspring of each female was classified by sex and phenotype. Statistical analysis were conducted with the BMDP Statistical Software which was implemented on the VAX Operating System.

\section{RESULTS}

The results are presented in table II. We have estimated some population parameters that characterize sperm predominance in double matings. $P_{2}$ is the proportion of second male offspring after remating (Boorman and Parker, 1976). $P_{2}^{w}$ is the weighted mean of $P_{2}$, equivalent to the mean of progeny proportions per female weighted by the female's total productivity. $P_{1}$ is the fraction of offspring sired by the first male and $P^{\prime}$ is the proportion of the first male's sperm that is used by a female before she remates (Gromko et al, 1984). $P^{\prime}$ was estimated from the control crosses as the weighted proportion of lst-3-day offspring over total female productivity. Both $P_{1}$ and $P_{2}$ were estimated from the female offspring, since the male offspring did not allow ascertainment of the sperm origin. The estimates of $P_{2}^{w}$ have been corrected for viability differences between the offspring of the 2 male genotypes (detected by the 3-way ANOVA; see below). In all cases, the values of $P_{2}^{w}$ were high. Groups $1 \mathrm{~b}$ and $2 \mathrm{~b}(2 \mathrm{nd}$ male w/Y) showed the lowest values, 0.91 and 0.92 respectively. The other 2 values (groups $1 \mathrm{a}$ and $2 \mathrm{a}$ ) were close to 1 . Moreover, only on the lst $\mathrm{d}$ after the 2 nd cross did we find offspring of the lst male. On the other hand, some of these descendants might have been produced prior to the time when the 2nd mating occurred. This would indicate that the actual $P_{2}$ values may be larger. The values of $P^{\prime}$, the fraction of sperm effectively used by the female before she remates, indicate the presence of at least $25-50 \%$ of sperm of the lst male when the 2 nd cross occurs. These values are underestimates, since after the 2nd cross was started females may lay eggs before remating. Given the high values of $P_{2}$, the bias of these underestimates is insignificant. The large values of $P_{2}$ suggest that the remaining sperm is not used in the following fertilizations. Since the $P_{2}$ 's variances are not equal, we used the Brown-Forsythe test (Dixon, 1985) to compare the $P_{2}$ values. The $P_{2}$ angular mean was used as the dependent variable. Differences were statistically significant between groups $1 \mathrm{a}, 2 \mathrm{a}$ and $1 \mathrm{~b}, 2 \mathrm{~b}$ (change in the order of males, $P<0.001$ ), but not between groups $1 \mathrm{a}, 1 \mathrm{~b}$ and $2 \mathrm{a}$, $2 \mathrm{~b}$ (change in the female genotype, $P=0.33$ ). So the variation in $P_{2}$ is a function of the male genotype. We find, therefore, a high degree of predominance of the last mated male, as well as possible selective differences in this component.

A possible source of error in the estimation of the $P_{2}$ values must be now considered. If during the time in which the 2 nd cross occurs a female remates more than once, then our $P_{2}$ values will be spurious, since they will correspond to $P_{2,3, \ldots, n}$, where $n$ is the number of times a female has remated from the begining of the experiment. Patterson and Stone (1952) found that the time between matings was $>135 \mathrm{~h}$ in this species. This, however, is not consistent with our results, since the percentage of females that remated was practically $100 \%$ (only 3 fertile females did not produce offspring from the 2 nd mate). Therefore, the time to remate in our population would be somewhat lower, but we do not know how much lower. 
Table II. Total female progeny and estimated values of parameters $P_{2}^{w}, P_{1}$ and $P^{\prime}$ for the groups described in table I. See text for their definitions. $n=$ No of females that produced progeny.

\begin{tabular}{|c|c|c|c|c|}
\hline \multicolumn{5}{|c|}{ Double-mated females } \\
\hline Group & $\begin{array}{l}\text { Total productivity } \\
\qquad \bar{x} \pm S E\end{array}$ & $\mathrm{P}_{2}^{w}$ & $P_{1}$ & $\mathbf{n}$ \\
\hline $1 \mathrm{a}$ & $139.3 \pm 11.7$ & 0.97 & 0.44 & 37 \\
\hline $1 \mathrm{~b}$ & $122.5 \pm 5.9$ & 0.91 & 0.62 & 35 \\
\hline $2 a$ & $382.9 \pm 18.8$ & 0.99 & 0.34 & 49 \\
\hline $2 \mathrm{~b}$ & $348.8 \pm 18.4$ & 0.92 & 0.44 & 46 \\
\hline \multicolumn{5}{|c|}{ Single-mated females } \\
\hline Group & $\begin{array}{l}\text { Total productivity } \\
\qquad \bar{x} \pm S E\end{array}$ & & $\mathrm{P}^{\prime}$ & $\mathrm{n}$ \\
\hline $3 a$ & $62.9 \pm 9.1$ & & 0.77 & 21 \\
\hline $3 \mathrm{~b}$ & $103.8 \pm 14.9$ & & 0.65 & 24 \\
\hline $4 \mathrm{a}$ & $221.3 \pm 21.8$ & & 0.50 & 22 \\
\hline $4 \mathrm{~b}$ & $229.4 \pm 17.1$ & & 0.66 & 25 \\
\hline
\end{tabular}

Wheeler (1947) found that $D$ buzzatii presents a high degree of expression of the insemination reaction. Patterson and Stone (1952) and Markow (1985) suggest that the insemination reaction works as a mechanism to preclude remating in females. Accordingly, $D$ buzzatii females would present a long refractory period before period remating, which would diminish the chances of a remating during the period of the 2nd cross. In this sense, $D$ buzzatii would be analogous to $D$ mojavensis, which delays additional matings (Markow, 1985). On the other hand, we have estimated the $P_{2}$ values in the case of females being remated 2 or 3 times. We have supposed that the proportion between $P_{i}$ and $P_{i-1}$ is $1: 2$. This assumption is based on the values of $P_{2}$ and $P_{3}$ found in $D$ hyde $i$ by Markow (1985). For females that remate twice the estimated values of $P_{2}$ for each group are: $P_{1 a, 2}=0.94, P_{1 b, 2}=0.84, P_{2 a, 2}=0.98$ and $P_{2 b, 2}=0.94$. For 3 times remated females they are: $P_{1 a, 2}=0.93, P_{1 b, 2}=0.81, P_{2 a, 2}=0.98$ and $P_{2 b, 2}=0.83$. These values are still large. So, we think that the $P_{2}$ values may be biased upwards, but not so much as to invalidate the conclusion that sperm predominance is high. However, the differences found in $P_{2}$ between male's genotypes may be due to differential mating success rather than to selection for sperm predominance.

Additionally, we performed a 3-way analysis of variance to confirm the previous comparisons (table III). The log of the progeny number sired by the first male was used as a dependent variable. The goal of this analysis was to test: a), the effect of the female remating; b), the effect of the female's genotype; and c), the effect of the male's genotype on the offspring of the lst male. We found significant fixed effects for each factor, but no interaction among them. First, the number of progeny sired by a male is significantly reduced if his partner subsequently remates $(F=9.42, P<0.01)$. From this we deduce again that sperm predominance occurs. 
Second, the female's genotype also influences productivity $(F=182.32, P<0.001)$ very significantly. These differences are probably due to the different genetic background of both stocks and not to the genetic marker itself. From the third factor, we find genetic differences among male genotypes $(F=10.95, P<0.01)$, but these cannot be attributed to selective differences in sperm predominance since the mate $\times$ male interaction is non-significant $(F=0.50, P=0.48)$. The absence of interaction indicates that the variance of this factor is negligible in relation to the variance due to other components, such as viability. It would appear, therefore, that selection for sperm predominance, if it exists, is not as important as the other components in these experiments.

Table III. A 3-way analysis of variance of the progeny number sired by the first mating male. The factors are mating type (mate), female genotype (female) and male genotype (male).

\begin{tabular}{lccrc}
\hline Source of variation & $d f$ & Miensquare & F & Probability \\
\hline Mating type (single or double) & 1 & 0.571 & 9.42 & 0.002 \\
Female genotype (w/w or w/+) & 1 & 11.057 & 182.32 & 0.000 \\
Male genotype (w/Y or +/Y) & 1 & 0.663 & 10.95 & 0.001 \\
Mate $\times$ female & 1 & 0.143 & 2.37 & 0.125 \\
Mate $\times$ male & 1 & 0.030 & 0.50 & 0.478 \\
Female $\times$ male & 1 & 0.072 & 1.19 & 0.276 \\
Mate $\times$ female $\times$ male & 1 & 0.039 & 0.65 & 0.420 \\
Error & 252 & 0.060 & & \\
\hline
\end{tabular}

Except for groups $1 b$ and $3 b$, statistically significant differences were found for the total progeny between the single and double mated females. Similar results have also been obtained in $D$ pseudoobscura (Turner and Anderson, 1983) and $D$ mojavensis (Markow, 1982), although contrary results have been reported for $D$ melanogaster ( $c f$ Boorman and Parker, 1976; Prout and Bundgaard, 1977; Gromko and Pyle, 1978; Alvarez and Fontdevila, 1981). In our case, it is clear that a single insemination is not sufficient to fertilize all the eggs a female can normally lay. This is against one assumption of the model of Prout and Bungaard (1977), which claims that female output is independent of the number of matings.

\section{DISCUSSION}

In the face of sperm competition, the male strategies can lead to 2 different, not necessarily exclusive, ways of adaptation. One which increases $P_{1}$, the fraction of offspring sired by the first male, either by an increase of $P^{\prime}$ (ie, through an increase in the time before female remating) or by "resisting" the predominance of the second male. The other way is to increase $P_{2}$. Our results seem to suggest that the male mating strategy of $D$ buzzatii is to maximize $P_{2}$ (ie the "remator" strategy) as well as $P^{\prime}$ (see Gwynne, 1984; p 141 for a possible selective explanation), whereas that of the female is multiple mating. In order to confirm this conclusion it would be necessary to perform different experiments with other stocks and varying the 
time of the second cross, since $P_{2}$ could depend both on the stock background and on the remating time (Boormer and Parker, 1976).

Gwynne (1984) believes that the males of those species with higher predominance supply food to the female or to his offspring. Markow and Ankney (1984, 1988) found in $D$ mojavensis that males transfer nutrients to females. We have indirect evidence that $D$ buzzatii males transfer yeast to females during mating (Starmer et al, 1988). Thus, transmission of nutrients might help to explain the increases in female productivity with the number of matings. A more likely possibility to account for female multiple mating is in relation with the high total productivity found in this species (Barker and Fredline, 1985; Barbadilla, 1986). Ridley (1988) reviews extensive evidence showing that species with higher productivities are less likely to receive sufficient sperms at one mating than species with low productivity, whereby in species with a high productivity a multiple mated female will leave more offspring than a single one.

The strong sperm predominance found in $D$ buzzatii has a considerable technical interest. Natural populations of this species show a moderately high inversion polymorphism in 2 autosomes. The karyotype of wild males can be ascertained by crossing them with virgin females from a laboratory stock homozygous for certain chromosome arrangements, and analyzing the salivary gland chromosomes of a number of larvae from each progeny. On the other hand, we seldom find out the karyotype of wild females, for they are usually already inseminated when collected in the field. One way to overcome this difficulty could be to remove sperm from the females by means of a low temperature treatment prior to their mating with the laboratory stock males (Anderson et al, 1979). This method, however, has proven to be completelly ineffective in $D$ buzzatii (unpublished results). The high values of $P_{2}$ suggest a simple solution to this problem. If wild females brought to the laboratory are crossed with males of known karyotypes and then transferred every other day to a new vial with fresh food, we expect that after a few days almost all the progeny will be sired by the laboratory males, allowing the correct identification of the female's karyotype. In a recent study carried out in the population of Carboneras (Ruiz et al, submitted) this prediction has proved to be essentially correct.

\section{ACKNOWLEDGMENTS}

We wish to thank 2 anonymous referees for their constructive comments on this manuscript. This work has been supported by grant No PB85-0071 from the Dirección General de Investigación Científica y Técnica (DGICYT, Spain), awarded to AF.

\section{REFERENCES}

Alvarez G, Fontdevila A (1981) Effect of the singed locus on the egg production curve of Drosophila melanogaster. Can J Genet Cytol 23, 327-336

Anderson WW, Levine L, Olvera O, Powel JR, de la Rosa ME, Salceda VM, Gaso MI, Guzmán J (1979) Evidence for selection by male mating success in natural populations of Drosophila pseudobscura. Proc Natl Acad Sci USA 76, 1519-1523 
Barbadilla A (1986) Estudio de los componentes tardíos de la fitness en una población experimental de Drosophila buzzatii. MS Thesis, Universidad Autónoma de Barcelona, Barcelona

Barker JSF, Fredline DK (1985) Reproductive biology of Drosophila buzzatii. Dros Inf Serv 61, 28-32

Boorman E, Parker GA (1976) Sperm (ejaculate) competition in Drosophila melanogaster, and the reproductive value of females to males in relation to female age and mating status. Ecol Entomol 1, 145-155

David J (1962) A new medium for rearing Drosophila in axenic conditions. Dros Inf Serv 36, 128

Dixon WJ (ed) (1985) BMDP Statistical Software. Manual University of California Press, CA, p 107

Gromko MH, Gilber DG, Richmond RC (1984) Sperm transfer and use in the multiple mating system of Drosophila. In: Sperm Competition and the Evolution of Animal Mating Systems (Smith RL, ed) Academic Press, NY, 371-426

Gromko MH, Pyle DW (1978) Sperm competition, male fitness, and repeated mating by female Drosophila melanogaster. Evolution 32, 588-593

Gwynne DT (1984) Male mating effort, confidence of paternity, and insect sperm competition. In: Sperm Competition and the Evolution of Animal Mating Systems (Smith RL, ed) Academic Press, NY, 117-149

Markow TA (1982) Mating systems of cactophilic Drosophila. In: Ecological Genetics and Evolution: the Cactus-Yeast Drosophila Model System (Barker JSF, Starmer WT, eds) Academic Press, Sidney, 273-287

Markow TA (1985) A comparative study of the mating system of Drosophila hydei. Anim Behav 33, 775-781

Markow TA, Ankney PF (1982) Drosophila males contribute to oogenesis in a multiple mating species. Science 224, 302-303

Markow TA, Ankney PF (1988) Insemination reaction in Drosophila: found in species whose males contribute material to oocytes before fertilization. Evolution 42, 1097-1101

Parker GA (1970) Sperm competition and its evolutionary consequences in the insects. Biol Rev 45, 525-567

Parker GA (1984) Sperm competition and the evolution of animal mating systems. In: Sperm Competition and the Evolution of Animal Mating Systems (Smith RL, ed) Academic Press, NY, 1-60

Patterson JT, Stone WS (1952) Evolution in the Genus Drosophila. Macmillan, NY, p 374

Prout T, Bundgaard J (1977) The population genetics of sperm displacement. Genetics 85, 95-124

Ridley M (1988) Mating frequency and fecundity in insects. Biol Rev 63, 509-549 Ruiz A, Santos M, Barbadilla A, Quezada-Díaz JE, Fontdevila A (1991) Genetic variance for body size in a natural population of Drosophila buzzati. Genetics (accepted for publication)

Ruiz A, Fontdevila A, Santos M, Seoane M, Torroja E (1986) The evolutionary history of Drosophila buzzatii. VIII. Evidence for endocyclic selection acting on the inversion polymorphism in a natural population. Evolution 40, 740-755 
Santos M, Ruiz A, Barbadilla A, Quezada-Díaz JE, Hasson E, Fontdevila A (1988) The evolutionary history of Drosophila buzzatii. XIV. Larger flies mate more ofter in nature. Heredity $61,255-262$

Santos M, Ruiz A, Fontdevila A (1989) The evolutionary history of Drosophila buzzatii. XIII. Random differentiation as a partial explanation of the observed chromosomal variation in a structured natural population. Am Nat 133, 183-197

Smith RL (ed) (1984) Sperm Competition and the Evolution of Animal Mating Systems. Academic Press, NY

Starmer WT, Peris F, Fontdevila A (1988) The transmission of yeast by Drosophila buzzatii during courtship and mating. Anim Behav 36, 1691-1695

Thornhill R, Alcock J (1983) The Evolution of Insect Mating Systems. Harvard Univ Press, Cambridge, MA

Turner ME, Anderson WW (1983) Multiple mating and female fitness in Drosophila pseudobscura. Evolution 37, 714-723

Turner ME, Anderson WW (1984) Sperm predominance among Drosophila pseudoobscura karyotypes. Evolution 38, 983-995

Wheeler MR (1947) The insemination reaction in intraspecific matings of Drosophila Univ Texas Publ Genet 4720, 78-115 